\title{
Use of telomelysin (OBP-301) in mouse xenografts of human head and neck cancer
}

\author{
OUMI NAKAJIMA ${ }^{1}$, DAIJU ICHIMARU ${ }^{2}$, YASUO URATA ${ }^{2}$, TOSHIYOSHI FUJIWARA $^{3}$, \\ TOMOHISA HORIBE $^{1}$, MASAYUKI KOHNO ${ }^{1}$ and KOJI KAWAKAMI ${ }^{1}$
}

\author{
${ }^{1}$ Department of Pharmacoepidemiology, Graduate School of Medicine and Public Health, Kyoto University, \\ Yoshidakonoe-cho, Sakyo-ku, Kyoto 606-8501; ${ }^{2}$ Oncolys Biopharma Inc., 3-16-33 Roppongi, Minato-ku, Tokyo 106-0032; \\ and ${ }^{3}$ Center for Gene and Cell Therapy, Okayama University Hospital, 2-5-1 Shikata-cho, Okayama 700-8558, Japan
}

Received April 29, 2009; Accepted July 14, 2009

DOI: $10.3892 /$ or_00000533

\begin{abstract}
We previously reported that telomerase-specific replication-component adenovirous, Telomelysin (OBP-301) has cytotoxic activity to the YCUT892, KCCT873, KCCT891, KCCL871, YCUM862, HN12, and KCCOR891 cell lines in vitro, and investigated the association between cytotoxic activity and adenoviral receptor expression. In this study, we evaluated the most appropriate way to administer telomelysin (OBP-301) in the treatment of squamous cell carcinoma of the head and neck (SCCHN), and assessed the effect of OBP301 in large subcutaneous KCCT873 human SCCHN tumors in immunodeficient mice. We also compared antitumor responses following three intratumoral (i.t.) injections of OBP-301 given daily, every 2 days or weekly. To investigate the mechanism of the antitumor effect, we evaluated cellular infiltration in treated tumors. OBP-301 showed remarkable antitumor activity against large KCCT873 tumors, and three treatment schedules produced similar antitumor effects. The weekly regimen also significantly reduced the growth of large tumors. Immunochemistry revealed that macrophages, but not natural killer cells, were responsible for tumor regression. A regimen of three weekly injections of OBP-301 has remarkable antitumor effects against large KCCT873 tumors. These results may provide a new platform for treating patients with localized SCCHN.
\end{abstract}

\section{Introduction}

Squamous cell carcinoma of the head and neck (SCCHN) accounts for $5 \%$ of newly diagnosed cancers in adults in the US and $8 \%$ of cancers worldwide (1). Most patients are

Correspondence to: Dr Koji Kawakami, Department of Pharmacoepidemiology, Graduate School of Medicine and Public Health, Kyoto University, Yoshida Konoecho, Sakyo-ku, Kyoto 606-8501, Japan

E-mail:kawakami-k@umin.ac.jp

Key words: telomelysin, OBP-301, telomerase, adenovirus, squamous cell carcinoma of the head and neck treated with various combinations of surgery, radiotherapy and systemic agents, but treatment fails in about half of patients (2). In light of the poor outlook for patients with recurrent disease, additional effective local-regional therapies are clearly required for the treatment of SCCHN. Conditionally replicating viruses targeted to tumors are being developed as a novel class of oncolytic agents. Oncolytic virotherapy has been evaluated in clinical trials of patients with SCCHN. For example, in a phase II trial, the addition of systemic cisplatin or 5-fluorouracil following direct intratumoral (i.t.) injection of the oncolytic adenovirus ONXY-015 resulted in clinical regression of SCCHN tumors (3).

Telomerase is a ribonucleoprotein complex responsible for the complete replication of chromosomal ends (4). It is expressed in $>85 \%$ of human cancers (5) but in only a few normal somatic cells (6). Telomerase activation is considered to be a critical step in carcinogenesis, and its activity is closely correlated to expression of human telomerase reverse transcriptase (hTERT) (7). hTERT can therefore be exploited as a cancer-specific promoter.

Telomelysin (OBP-301) is a telomerase-specific replicationcompetent adenovirus vector in which the hTERT promoter element drives the expression of $E 1 A$ and $E 1 B$ genes, which are linked to an internal ribosome entry site $(8,9)$. Telomelysin has been shown to selectively kill human cancer cells (8-11). In infected human tumor cells in vitro, OBP-301 replication produces the endogenous danger-signaling molecule uric acid, which in turn stimulates dendritic cells to produce interferon $\gamma(\mathrm{IFN} \gamma)$ and interleukin (IL)-12 (12). In vivo, OBP-301 induces $\mathrm{CD}^{+}$and $\mathrm{CD}^{+} \mathrm{T}$ cells following production of INF $\gamma$ (13).

A phase I study of OBP-301 has been started in the US in patients with various types of progressive solid cancer, including SCCHN (14). We have previously reported that a 5-day i.t. administration of OBP-301 to nude mice bearing subcutaneous KCCT873 human SCCHN xenografts had significant antitumor activity (15). In the present study, we extend this work by assessing the antitumor activity of OBP301 in advanced SCCHN tumors. We also compared different treatment schedules, in order to explore the likely use of OBP-301 in a clinical setting, and performed immunohistochemistry to evaluate the mechanism of the antitumor response. 


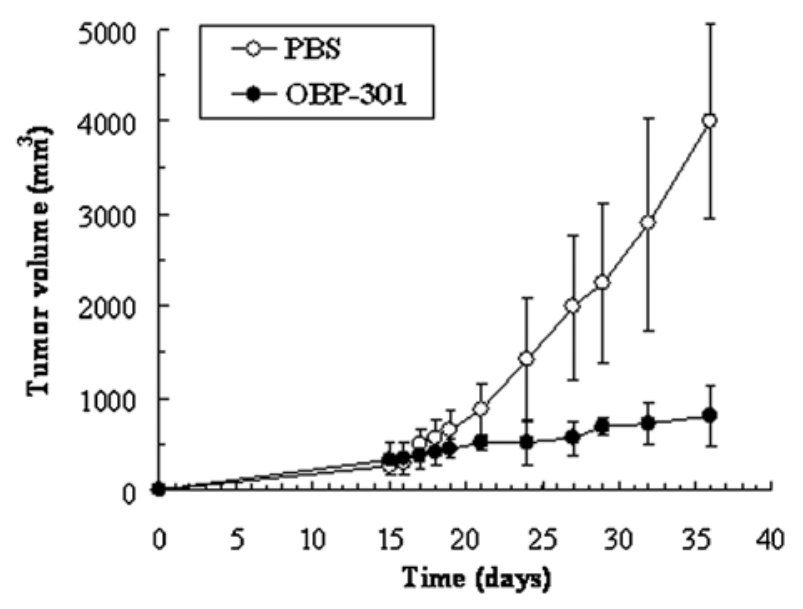

Figure 1. Growth of large SCCHN tumors following intratumoral injection of OBP-301 ( $3 \times 10^{10}$ v.p.) daily for 5 days from day 15 to day 19 after inoculation of KCCT873 cells. Tumors had an average volume of $298 \pm 33 \mathrm{~mm}^{3}$ at the start of treatment. Control mice were given injections of vehicle. Values are means $\pm \mathrm{SD}$ ( $n=7$ mice per group).

\section{Materials and methods}

Adenovirus. OBP-301 was provided by Oncolys Biopharma Inc. (Tokyo, Japan). The ratio of virus particle (v.p.) titer to infection titer (plaque-forming units) was 51:1.

Cell culture. The KCCT873 (16) human SCCHN cell line was cultured in RPMI-1640 (Nacalai Tesque, Kyoto, Japan) containing $10 \%$ fetal bovine serum, $1 \mathrm{mM}$ HEPES, $100 \mu \mathrm{g} /$ $\mathrm{ml}$ penicillin and $100 \mu \mathrm{g} / \mathrm{ml}$ streptomycin. Cells $\left(3 \times 10^{6}\right.$ cells per $150-\mathrm{mm}$ dish) were incubated for 3 days before injection, at $37^{\circ} \mathrm{C}$ in an atmosphere containing $5 \% \mathrm{CO}_{2}$.

Xenograft model. Female athymic nude mice (BALB/c nu/nu), aged 5-6 weeks, were obtained from SLC Inc. (Hamamatsu, Japan). Animal care and experiments were in accordance with the guidelines of the Kyoto University School of Medicine. The mice were allowed to become acclimatized for 1 week in a sterile environment and were maintained at $24^{\circ} \mathrm{C}$ and $50 \%$

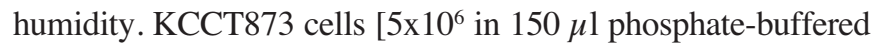
saline (PBS)] were injected subcutaneously into the flanks of nude mice. Palpable tumors developed within 5 days. Tumors were measured using Vernier calipers. Tumor volume on a given day was calculated using the formula: $1 / 2$ [(tumor length) $\mathrm{x}$ (tumor width $)^{2}$.

Antitumor effect of OBP-301 in large tumors. Tumors were allowed to grow to a mean volume of $298 \pm 33 \mathrm{~mm}^{3}$ (day 15). Mice were then given OBP-301 ( $3 \times 10^{10}$ v.p. in a volume of $30 \mu 1)$ i.t. daily for 5 days. Control mice were given i.t. injections of PBS. Our previous studies had shown that this titer had marked antitumor effects.

Optimization of OBP-301 treatment schedule. To assess the effect of the treatment schedule with OBP-301, mice were given three i.t. injections of OBP-301 $\left(3 \times 10^{10} \mathrm{v} . \mathrm{p}\right.$. in a volume of $30 \mu 1$ ), which were administered every day (q.d., on days 5,6 and 7 after tumor inoculation, when mean tumor volume was $39 \mathrm{~mm}^{3}$ ), every 2 days (q.o.d., on days 5, 7 and 9), or once

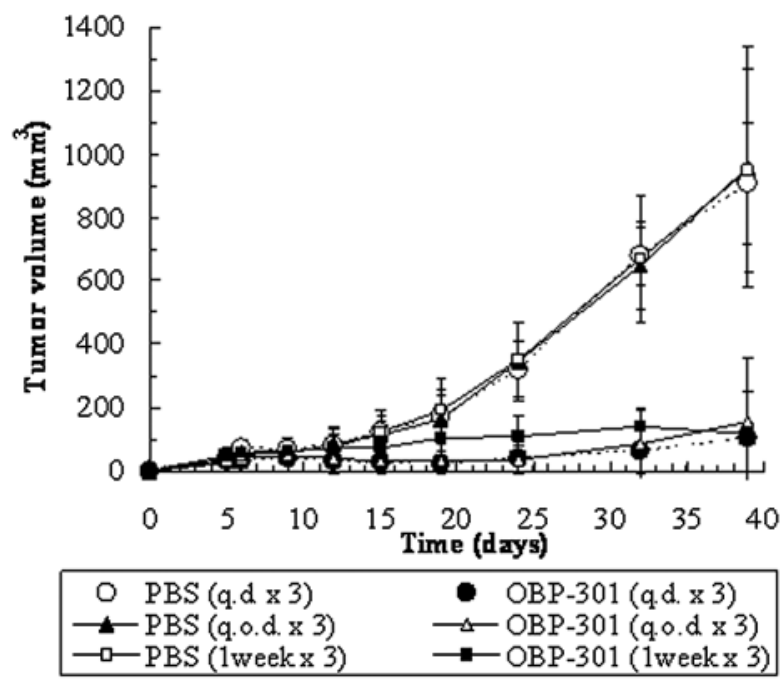

Figure 2. Growth of KCCT873 tumor xenografts in nude mice following three intratumoral injections of OBP-301 given daily (q.d., days 5, 6 and 7 after tumor inoculation), every 2 days (q.o.d., days 5, 7 and 9), or weekly (days 5, 12 and 19) compared with controls (which received injections of vehicle). Values are means $\pm \mathrm{SD}(\mathrm{n}=6$ mice per group). Similar results were obtained in a second experiment.

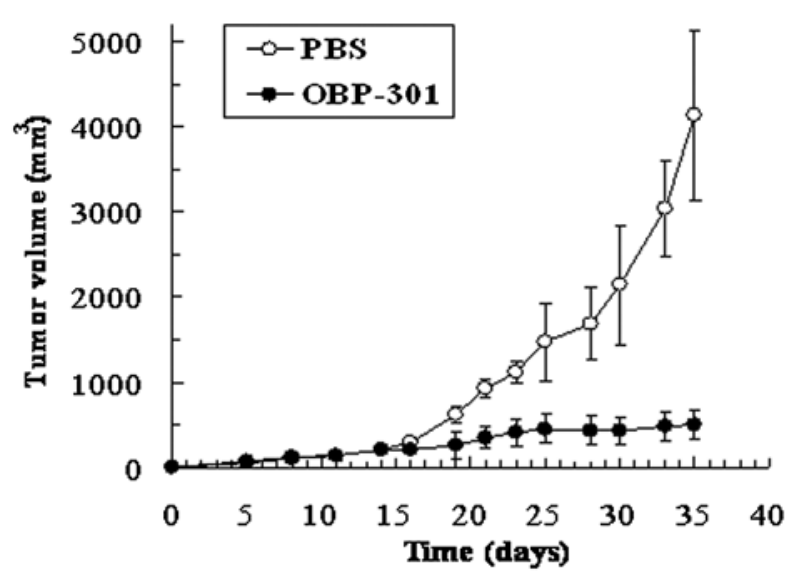

Figure 3. Antitumor activity of OBP-301 in large KCCT873 tumors. Nude mice with established KCCT873 tumors were given three i.t. injections of OBP-301 (9x10 10 v.p) or vehicle on days 14, 21 and 28 after tumor inoculation. Values are means $\pm \mathrm{SD}$ ( $\mathrm{n}=6$ mice per group). Similar results were obtained in a second experiment.

a week (days 5, 12 and 19). The weekly regimen was also tested in mice bearing large established KCCT873 tumors, starting 14 days after tumor implantation, when mean volume had reached $204 \pm 3 \mathrm{~mm}^{3}$. Mice were given i.t. injections of OBP-301 (9x10 10 v.p.) on days 14, 21 and 28. A larger titer was used in these experiments, as preliminary studies had shown that $3 \times 10^{10} \mathrm{v} . \mathrm{p}$. did not induce tumor regression in these larger tumors.

Immunohistochemistry. To investigate the mechanism of the antitumor response induced by OBP-301, we examined cellular infiltration in subcutaneous KCCT873 human SCCHN tumors in immunodeficient mice. These mice were given a single i.t. injection of OBP-301 (9x10 $10^{10}$ v.p.) 14 days after tumor inoculation. Tumors were excised on days 1, 7, 14 and 21 

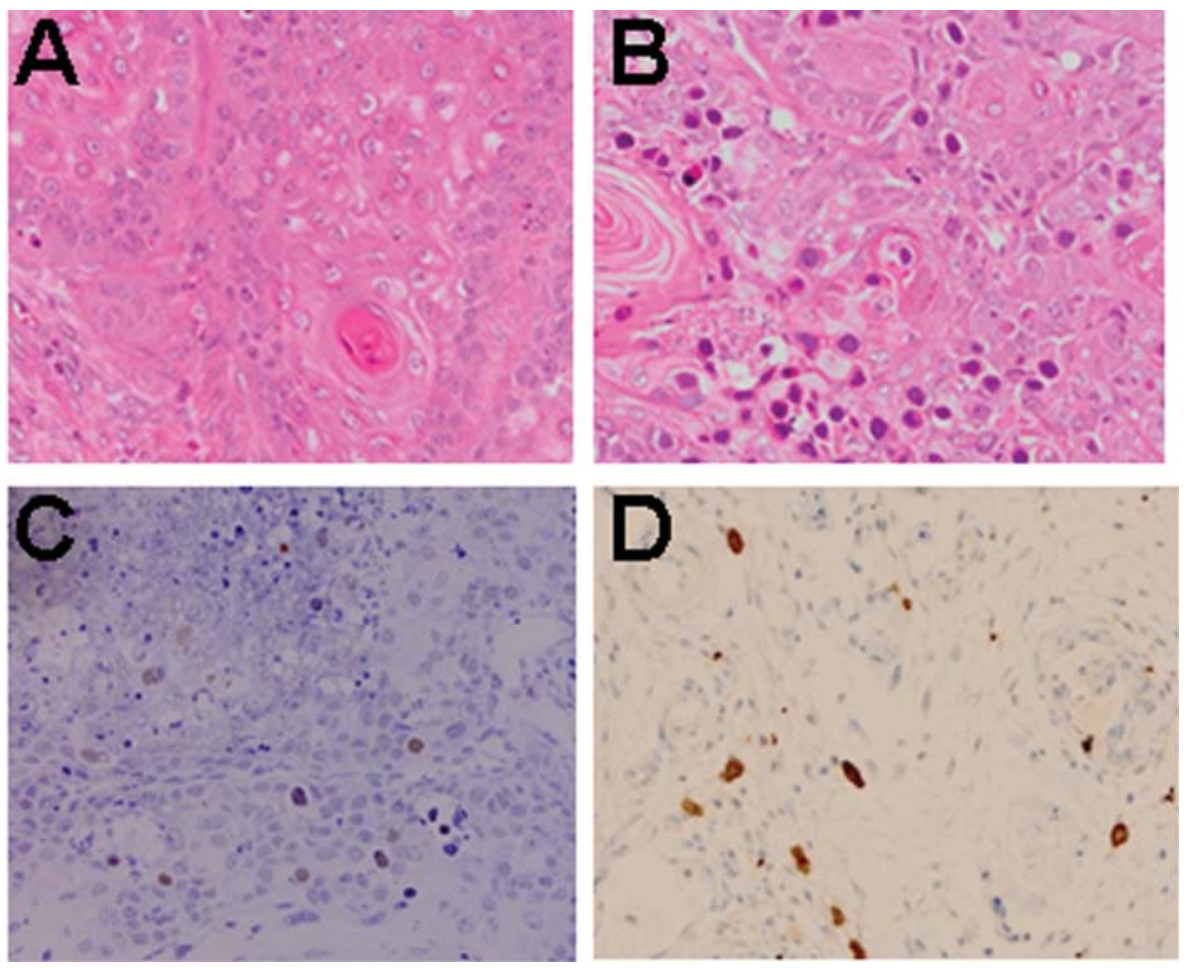

Figure 4. Effects of OBP-301 treatment on tumor histology. Mice with established KCCT873 tumors were given a single intratumoral injection of OBP-301 $\left(9 \times 10^{10}\right.$ v.p.). The images show sections stained with hematoxylin and eosin from control (A) and treated (B) mice (both $\left.\mathrm{x} 200\right)$ and sections from treated mice labeled with antibodies to murine macrophages (C) or natural killer cells (D; both $\mathrm{x} 400$ ).

after administration of OBP-301 and immediately fixed in $10 \%$ formalin. Sections $(5 \mu \mathrm{m})$ of the paraffin-embedded tissues were prepared and analyzed by immunohistochemistry as described previously (17). Slides were incubated with antibodies against murine macrophages (F4/80) or natural killer (NK) cells (NK 1.1; both Caltag Laboratories, Burlingame, CA) or isotype control for $18 \mathrm{~h}$ at $4^{\circ} \mathrm{C}$. The sections were counterstained with hematoxylin and eosin. Immunohistochemical assays were performed twice and showed similar results.

Statistical analysis. Data are presented as mean \pm SD. Differences in tumor growth between treatment and control groups were analyzed by the Student's t-test. $\mathrm{P}<0.05$ was considered significant.

\section{Results}

Effect of OBP-301 on large SCCHN tumors. Treatment started when tumors had reached a mean volume of $298 \pm 33 \mathrm{~mm}^{3}$ (day 15). The control tumors grew rapidly, reaching $4001 \mathrm{~mm}^{3}$ by day 36 , whereas tumors treated with OBP-301 showed less growth during the treatment period (Fig. 1): by day 36, mean tumor volume in the treated group was $806 \mathrm{~mm}^{3}$, which was significantly smaller than the controls $(\mathrm{P}<0.001)$. Complete tumor regression did not occur but OBP-301 clearly mediated antitumor effects in large SCCHN tumors.

Optimization of OBP-301 treatment schedule. To assess the effect of treatment schedule on tumor growth, mice were given three i.t. injections of OBP-301, q.d., q.o.d. or weekly, starting on day 5 after tumor inoculation. As shown in Fig. 2, i.t. administration of OBP-301 showed considerable antitumor activity in all groups. The mean tumor volume in the q.d. group was $105 \mathrm{~mm}^{3}$ at day 39, which was $88 \%$ smaller than that in control tumors $\left(909 \mathrm{~mm}^{3}\right)(\mathrm{P}<0.005)$. Four of the seven tumors had regressed completely by day 24 , although two of these tumors had re-appeared and started to grow again by day 39. The mean tumor volume in the q.o.d. group was $156 \mathrm{~mm}^{3}$ at day 39 , which was $84 \%$ smaller than control tumors $\left(959 \mathrm{~mm}^{3}\right.$; $\mathrm{P}<0.001)$. Tumors disappeared completely in two of the seven mice by day 39. Marked antitumor activity was also seen in animals given i.t. injections of OBP-301 at weekly intervals: the mean tumor volume on day 39 was $118 \mathrm{~mm}^{3}$, which was $88 \%$ smaller than control tumors $\left(947 \mathrm{~mm}^{3} ; \mathrm{P}<0.001\right)$. These results show that three i.t. injections of OBP-301 effectively reduced KCCT873 SCCHN tumor growth, regardless of the interval between injections. Since one injection per week provided significant antitumor activity, we also assessed this regimen in animals bearing large established KCCT873 tumors. Mice were given i.t. injections of OBP-301 $\left(9 \times 10^{10}\right.$ v.p.) on days 14, 21 and 28 after tumor innoculation. Mean tumor volume on day 14 was $204 \mathrm{~mm}^{3}$. Tumors of treated mice continued to grow slowly during the treatment period but mean tumor volume on day 35 was significant smaller $\left(501 \mathrm{~mm}^{3}\right)$ than in control mice $\left(4133 \mathrm{~mm}^{3} ; \mathrm{P}<0.001\right)$ (Fig. 3). These results show that larger SCCHN tumors can be successfully treated with OBP-301 using a regimen of one injection per week.

Infiltration of immune cells into tumor site after OBP-301 treatment. To investigate the mechanism of antitumor response 
induced by OBP-301 treatment, we examined treated tumors for cellular infiltration $1,7,14$ and 21 days after administration of a single i.t. injection of OBP-301 (9x10 10 v.p.). No cellular infiltration was seen in tumors from control mice (Fig. 4A). Several necrotic areas were seen in sections of dying tumor 1 and 7 days after OBP-301 treatment (Fig. 4B). These areas were surrounded by fibrotic elements. Interestingly, a number of phagocytes, most resembling monocytes morphologically, were seen in necrotic areas and in viable tumor areas. We then performed immunohistochemical staining to determine the characteristics of inflammatory cells. As shown in Fig. 4C and $\mathrm{D}$, moderate numbers of macrophage-marker-positive cells (Fig. 4C), and NK-marker-positive cells (Fig. 4D) were seen. Tumors treated with OBP-301 showed localization of macrophages in the tumors 7 days after injection but these cells had disappeared 14 days after OBP-301 treatment (data not shown). Since the number of NK cells was not changed dramatically in treated or control tumors, these results suggest that macrophage infiltration, but not NK cells, are responsible for the tumor regression induced by OBP-301.

\section{Discussion}

We recently reported that three or five i.t. injections of OBP-301 dramatically inhibited the growth of KCCT873 tumors in vivo (15). The present study adds to the antitumor profile of OBP-301 in SCCHN tumor-bearing mice, showing that OBP-301 has antitumor activity against large tumors.

Three i.t. injections of OBP-301 showed pronounced antitumor activity in KCCT873 tumors, regardless of whether the injections were given q.d., q.o.d. or weekly. The weekly treatment regimen also had marked antitumor effects in large KCCT873 tumors. These results show that OBP-301 is likely to provide therapeutic benefit in localized SCCHN tumors even when tumors are large. However, to achieve complete regression of large tumors patients may require OBP-301 in combination with conventional chemotherapy.

Histological examination showed that macrophages were present in regressing tumors 1 and 7 days after OBP-301 treatment. Thus, OBP-301 treatment seems to enhance the host immune response at the tumor site, thereby eliminating residual tumor cells that have escaped the cytotoxic effects of OBP-301. Endo and colleagues reported that, in vitro, replication of OBP-301 produced the endogenous dangersignaling molecule, uric acid, in infected human tumor cells, which in turn stimulated dendritic cells to produce INF $\gamma$ and IL-12 (12). Fujita and colleagues reported that OBP-301 induced $\mathrm{CD}^{+}$and $\mathrm{CD}^{+} \mathrm{T}$ cells following production of INF $\gamma$ in immune-competent mice (13). Taken together with our current results obtained in athymic nude mice, it is suggested that the effect of OBP-301 against SCCHN tumors might involve immune responses in addition to direct effects on cancer cells.

In this study, we demonstrated that OBP-301 exerts a strong antitumor effect against human SCCHN tumors and may therefore be a promising tool for virotherapy. Generally, to improve the therapeutic index, there is a need for antitumor agents that selectively target tumors and spare normal tissues, and many investigators have been working to develop such cancer-specific biochemical drugs. To achieve this tumor specificity, OBP-301 was developed from a replicationselective tumor-specific oncolytic adenovirus. OBP-301 has been shown to be effective against human cancers $(8,9,15)$. A phase I study has been initiated in the US to assess the safety and tolerability of OBP-301 in patients with various types of progressive solid cancers, including SCCHN. Fujiwara and colleagues reported that nine patients received escalating dose levels of OBP-301 ( $1 \times 10^{10}$ to $1 \times 10^{12}$ v.p.) as monotherapy had stable disease at day 28 of assessment, although 6 patients showed 6.6 to $34 \%$ tumor size reduction (14). Results from current clinical trials should yield additional information on its efficacy. In this study, i.t. injections of OBP-301 showed significant antitumor activity in SCCHN tumors, regardless of the interval between injections. These results suggest that OBP-301 can be used as outpatient treatment, once a week in clinic. OBP-301 is thought to be a treatment that may be possible to administer long-term in a weekly regimen with a low invasiveness. The preliminary information obtained from our study will be useful for the planning of future clinical trials of OBP-301 in SCCHN.

In conclusion, this study clearly demonstrates that a regimen of three weekly injections of OBP-301 has remarkable antitumor effects in vivo against large KCCT873 SCCHN tumors. These findings suggest that the replication-selective oncolytic virus provides a new platform for treating patients with localized SCCHN.

\section{Acknowledgements}

We thank Ritsuko Asai (Department of Pharmacoepidemiology, Kyoto University) for technical assistance with tissue culture. This work was supported in part by a research grant-in-aid from the Japanese Ministry of Health, Labour and Welfare to Koji Kawakami (KH33332).

\section{References}

1. Jemal A, Siegel R, Ward E, Murray T, Xu J, Smigal C and Thun MJ: Cancer statistics, 2006. CA Cancer J Clin 56: 106-130, 2006.

2. Cohen EE, Lingen MW and Vokes EE: The expanding role of systemic therapy in head and neck cancer. J Clin Oncol 22: 1743-1752, 2004

3. Shin EJ, Wanna GB, Choi B, et al: Interleukin-12 expression enhances vesicular stomatitis virus oncolytic therapy in murine squamous cell carcinoma. Laryngoscope 117: 210-214, 2007.

4. Blackburn EH: Structure and function of telomeres. Nature 350: 569-573, 1991.

5. Kim NW, Piatyszek MA, Prowse KR, et al: Specific association of human telomerase activity with immortal cells and cancer. Science 266: 2011-2015, 1994.

6. Shay JW and Wright WE: Telomerase activity in human cancer. Curr Opin Oncol 8: 66-71, 1996.

7. Nakayama J, Tahara H, Tahara E, et al: Telomerase activation by hTERT in human normal fibroblasts and hepatocellular carcinomas. Nat Genet 18: 65-68, 1998.

8. Kawashima T, Kagawa S, Kobayashi N, et al: Telomerasespecific replication-selective virotherapy for human cancer. Clin Cancer Res 10: 285-292, 2004.

9. Umeoka T, Kawashima T, Kagawa S, et al: Visualization of intrathoracically disseminated solid tumors in mice with optical imaging by telomerase-specific amplification of a transferred green fluorescent protein gene. Cancer Res 64: 6259-6265, 2004.

10. Taki M, Kagawa S, Nishizaki M, et al: Enhanced oncolysis by a tropism-modified telomerase-specific replication-selective adenoviral agent OBP-405 ('Telomelysin-RGD'). Oncogene 24: 3130-3140, 2005. 
11. Wickham TJ, Mathias P, Cheresh DA and Nemerow GR: Integrins alpha $\mathrm{v}$ beta 3 and alpha $\mathrm{v}$ beta 5 promote adenovirus internalization but not virus attachment. Cell 73: 309-319, 1993.

12. Endo Y, Sakai R, Ouchi M, et al: Virus-mediated oncolysis induces danger signal and stimulates cytotoxic T-lymphocyte activity via proteasome activator upregulation. Oncogene 27: 2375-2381, 2008.

13. Fujita K, Kimura M, Kondo N, et al: Anti-tumor effects of telomelysin for head and neck squamous cell carcinoma. Oncol Rep 20: 1363-1368, 2008.

14. Fujiwara T, Tanaka N, Nemunaitis J, et al: Phase I trial of intratumoral administration of OBP-301, a novel telomerasespecific oncolytic virus, in patients with advanced solid cancer: Evaluation of biodistribution and immune response. Proc ASCO. J Clin Oncol 26: abs. 3572, 2008.
15. Nakajima O, Matsunaga A, Ichimaru D, Urata Y, Fujiwara Y and Kawakami K: Telomerase-specific virotherapy in an animal model of human head and neck cancer. Mol Cancer Ther 8: $171-177,2009$

16. Kawakami K, Leland P and Puri RK: Structure, function, and targeting of interleukin 4 receptors on human head and neck cancer cells. Cancer Res 60: 2981-2987, 2000.

17. Kawakami K, Kawakami M, Husain SR and Puri RK: Targeting interleukin-4 receptors for effective pancreatic cancer therapy. Cancer Res 62: 3575-3580, 2002. 